\title{
La tradition italienne des manuscrits d'auteur : un patrimoine préservé et une culture littéraire
}

\section{Christian Del Vento et Pierre Musitelli}

\section{(2) OpenEdition}

1 Journals

Édition électronique

URL : https://journals.openedition.org/genesis/4298

DOI : 10.4000/genesis.4298

ISSN : 2268-1590

Éditeur :

Presses universitaires de Paris Sorbonne (PUPS), Société internationale de génétique artistique littéraire et scientifique (SIGALES)

Édition imprimée

Date de publication : 15 décembre 2019

Pagination : 7-12

ISBN : 979-10-231-0650-3

ISSN : 1167-5101

\section{Référence électronique}

Christian Del Vento et Pierre Musitelli, « La tradition italienne des manuscrits d'auteur : un patrimoine préservé et une culture littéraire », Genesis [En ligne], 49 | 2019, mis en ligne le 15 décembre 2019, consulté le 03 septembre 2021. URL : http://journals.openedition.org/genesis/4298 ; DOI : https:// doi.org/10.4000/genesis.4298

Ce document a été généré automatiquement le 3 septembre 2021.

Tous droits réservés 


\title{
La tradition italienne des manuscrits d'auteur : un patrimoine préservé et une culture littéraire
}

\author{
Christian Del Vento et Pierre Musitelli
}

\section{Une situation d'exception}

1 Rien ne justifierait en apparence la parution d'un numéro de Genesis consacré de façon exclusive à une tradition littéraire "nationale», dans la mesure où l'on peut difficilement imaginer qu'un écrivain italien, confronté à une page blanche, soit soumis à des phénomènes cognitifs radicalement différents par rapport à ses collègues français, espagnols, anglais ou allemands. Force est pourtant de constater qu'au-delà même de la spécificité linguistique des dossiers de genèse ici étudiés, la tradition littéraire italienne constitue d'un point de vue génétique, par ses spécificités propres, un cas exceptionnel à plusieurs égards. Par sa précocité, tout d'abord, elle invite la critique génétique à se mesurer à des manuscrits bien antérieurs au siècle des Lumières $^{1}$. La monumentale entreprise des Autografi dei letterati italiani, dirigée par Emilio Russo et Matteo Motolese ${ }^{2}$, qui s'inscrit dans la droite ligne de l'Iter italicum de Paul Oskar Kristeller $^{3}$ et dont une présentation figure dans la section des «Chroniques» de ce numéro, témoigne avec évidence de l'extraordinaire richesse des archives littéraires italiennes du Moyen Âge et de la première Modernité. Il suffit de parcourir les volumes déjà publiés pour constater l'accroissement du nombre de documents autographes qui nous sont parvenus : 156 manuscrits et 50 livres annotés pour 26 écrivains des XIII $^{\mathrm{e}}$ et XIV ${ }^{\mathrm{e}}$ siècles ; 706 manuscrits et 424 livres annotés pour 26 écrivains du xv ${ }^{\mathrm{e}}$ siècle; 1936 manuscrits et 748 livres annotés pour 61 écrivains du XvI ${ }^{\mathrm{e}}$ siècle. L'ouvrage n'est pas encore achevé, mais il donne déjà une idée précise du nombre de manuscrits d'auteur conservés depuis l'origine de cette tradition, qui est née, rappelons-le, sous le signe de l'autographie, puisque le plus ancien document 
littéraire italien retrouvé à ce jour, la chanson Quand eu stava, datée du dernier quart du $\mathrm{XII}^{\mathrm{e}}$ siècle, semble être un manuscrit d'auteur.

Outre la précocité de la conservation des archives, le caractère exceptionnel du cas italien tient, aux yeux du généticien, à la longue tradition de valorisation et d'étude de ces manuscrits. Comme le montre ici Christian Del Vento, c'est en Italie que l'interprétation littéraire commence à s'intéresser pour la première fois, dans le deuxième quart du XvI ${ }^{\mathrm{e}}$ siècle, aux brouillons des auteurs. C'est également en Italie que voit le jour la "critique des variantes ", véritable ancêtre de la critique génétique, et que parait, en 1642, la toute première édition "génétique »: celle des brouillons de Pétrarque $^{4}$. Les écrivains commencent à explorer, en apprentis, l'atelier des grands auteurs de la tradition dont les manuscrits apparaissent comme de précieux outils pédagogiques : l'étude génétique se double d'une démarche poétique.

C'est en outre en Italie que prend forme, dès la deuxième moitié du XIv $v^{e}$ siècle, la "fonction auteur ", cette conception nouvelle du rapport entre l'homme de lettres et ses propres écrits qui ne s'affirmera dans d'autres traditions littéraires qu'à partir de la fin du XVII siècle. Les écrivains deviennent les premiers responsables de la préservation de leurs archives littéraires et de leur bibliothèque. Pour nombre d'entre eux, il s'agit de défendre leur œuvre et d'en garantir l'authenticité. Comme l'écrit Claude Cazalé Bérard dans l'étude qu'elle consacre aux manuscrits de Boccace, ce sont les écrivains eux-mêmes qui, dès le Moyen Âge, " posent explicitement - [...] avec une anticipation extraordinaire - le problème du statut du texte, de l'auteur, des enjeux de ce qui avec eux va devenir la littérature ».

Si les archives littéraires font l'objet d'un intérêt croissant au cours de la Renaissance, c'est aussi parce qu'elles doivent permettre en Italie la définition d'une norme linguistique commune au sein d'une aire géographique et culturelle relativement homogène mais dépourvue, du fait de son histoire, d'un centre politique capable d'imposer sur l'ensemble du territoire son hégémonie culturelle. L'étude des manuscrits autographes et des variantes d'auteur restitue, dans sa pureté et dans son authenticité, le travail sur la langue mené par les grands écrivains et devient ainsi, et avant tout, un instrument d'apprentissage des techniques de rédaction et d'un «bon usage " grammatical et linguistique pour les aspirants lettrés. Tel fut le postulat de Pietro Bembo (1470-1547), premier homme de lettres à étudier les brouillons de Pétrarque et à s'en servir dans ses Prose della volgar lingua $(1525)^{5}$ pour définir une norme linguistique commune qui allait avoir force de loi pour les trois siècles à venir. Telles furent aussi les raisons de l'importance très tôt conférée en Italie aux formes de la représentation éditoriale et typographique, appelée à reproduire les manuscrits d'auteur pour en valoriser les aspects linguistiques et stylistiques. Ces avancées servirent en outre une entreprise lexicographique pionnière en Europe : le dictionnaire de l'Académie de la Crusca (1612), fondé sur le dépouillement des grands auteurs notamment les Florentins du XIV ${ }^{\mathrm{e}}$ siècle, mais aussi Machiavel, Bembo et l'Arioste - et destiné à devenir un instrument indispensable pour tous ceux qui entendaient écrire dans un « bon » italien.

La préservation de cet exceptionnel patrimoine archivistique nourrit sur la longue durée l'intérêt philologique, la bibliophilie, voire la vénération des écrivains italiens pour les grands textes du passé. La figure 1 en offre un exemple évocateur : lorsque le jeune Vittorio Alfieri, âgé de trente-quatre ans, consulte à Ferrare les brouillons du Roland furieux, il laisse au crayon, au bas de la page, la trace de son pèlerinage 
poétique : «Vittorio Alfieri vide e venerò, 18 giugno 1783 » («Vittorio Alfieri vit et vénéra, 18 juin $1783 »)$.

Fig. 1 : Page manuscrite de l'Arioste annotée par Alfieri.

(c) Ferrare, Biblioteca Comunale Ariostea, ms Cl. I, A, It. 1, f. 53 v. Su concessione del Ministero dei beni e delle attività culturali e del turismo. Divieto di ulteriore riproduzione o duplicazione con qualsiasi mezzo.

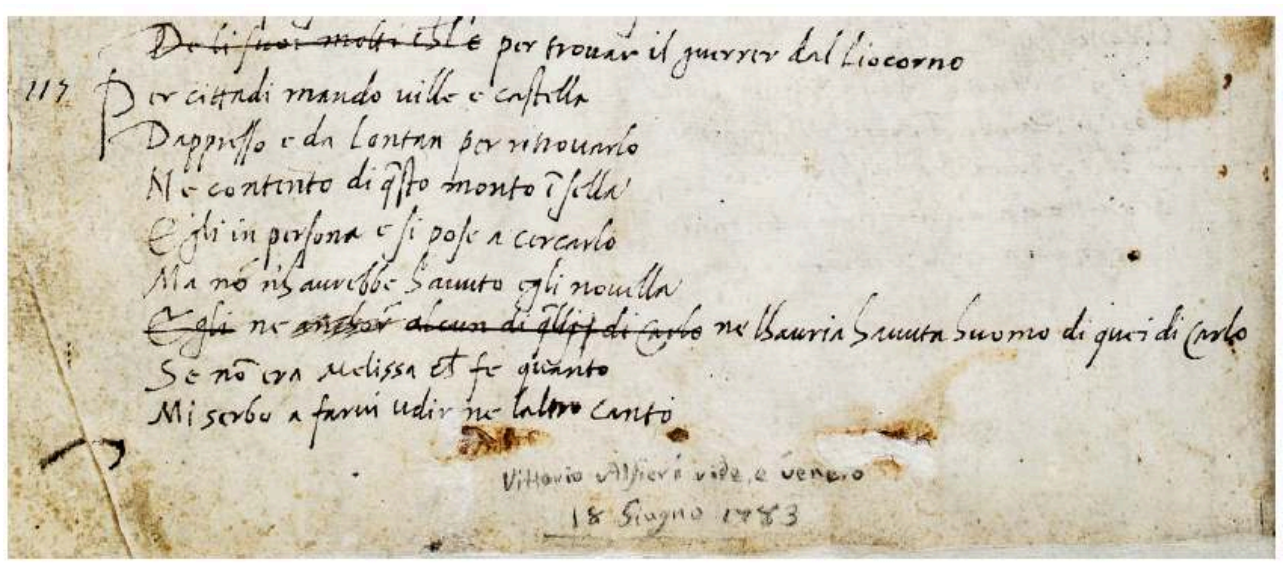

Le cas italien finit toutefois par perdre sa spécificité au cours du XIX siècle. Le regard que la culture transalpine porte sur les manuscrits des écrivains rejoint alors celui d'autres traditions littéraires. À mesure que se concrétise le projet d'unification politique de la péninsule, l'idée se fait jour que la tradition littéraire n'est plus une source de légitimation linguistique et qu'il devient nécessaire de fonder une langue littéraire actuelle, vivante, capable d'exprimer la totalité des besoins d'une nation moderne. La genèse mouvementée des Fiancés $(1821,1827$ et 1840) d'Alessandro Manzoni (1785-1873), que retrace Giulia Raboni, témoigne de cet éloignement progressif vis-à-vis des modèles de la tradition.

Dans ce contexte nouveau, l'intérêt pour les manuscrits d'auteur s'estompe au tournant du $\mathrm{xx}^{\mathrm{e}}$ siècle, en pleine mouvance néo-idéaliste. Benedetto Croce (1866-1952), qui exerça un haut magistère sur la culture italienne au cours de la première moitié $d u x^{e}$ siècle et au-delà, considérait l'œuvre littéraire comme une " perfetta forma fantastica ", c'est-à-dire comme le fruit, non intentionnel, d'une intuition. Le texte ne peut donc s'apprécier qu'en tant que forme pure et immuable, isolée de son processus de création et détachée de tout lien avec le monde contingent. C'est pourtant la philosophie et l'esthétique de Croce qui déterminèrent en partie le contexte dans lequel se manifesta à la fin des années 1930 un regain d'intérêt pour les manuscrits d'auteur et pour l'approche génétique des archives d'écrivains. Gianfranco Contini (1912-1990), élève à Paris de Joseph Bédier, sut mettre à profit la leçon de Mallarmé et, surtout, de Paul Valéry, pour se faire l'artisan d'une renaissance de la "critique des variantes ", après les expériences pionnières $d u x I^{e}$ siècle. Bien que Contini fût partagé entre une adhésion à la pensée de Croce et la volonté de s'opposer à son hégémonie, comme le montrent les contributions de Simone Albonico et de Paola Italia, il se retrouva dans l'immédiat après-guerre au centre d'un vif débat qui est à l'origine de la génétique italienne et du regard nouveau que la culture italienne contemporaine porte sur les manuscrits d'auteur. Ce contexte détermina les orientations d'une grande partie de la critique littéraire en Italie au cours de la deuxième moitié du xxe siècle. 


\section{Un parcours entre monuments littéraires et corpus exemplaires}

$8 \mathrm{Si}$, comme nous l'avons mentionné, le cas italien est extraordinairement précoce, ce n'est pas seulement au regard de documents tels que les manuscrits autographes du Chansonnier de Pétrarque ou du Décaméron de Boccace, qui relèvent de la catégorie de ce que Roger Chartier a nommé les "livres d'auteurs $»^{6}$. C'est aussi en raison de la conservation de matériaux génétiques d'une autre nature, comme les brouillons $\mathrm{du}$ Chansonnier (le fameux Codice degli abbozzi, connu du public français ${ }^{7}$, et qui témoigne du travail de rédaction auquel Pétrarque se consacra pendant de longues années), les manuscrits préparatoires du Roland furieux, les Zibaldoni de Boccace et les livres annotés de la bibliothèque du Tasse. C'est précisément dans l'atelier de Boccace, de l'Arioste et dans la bibliothèque du Tasse que ce numéro de Genesis se propose d'accompagner le lecteur afin de lui présenter quelques cas exemplaires de corpus manuscrits d'écrivains italiens et de questionner, à travers eux, les processus de la création littéraire.

9 «Livre-archives d'auteur », selon la formule d'Armando Petrucci, véritables répertoires de documents de genèse, les Zibaldoni de Boccace dévoilent lo scrittoio, la table de travail d'un auteur dont l'œuvre ne se limita pas au seul Décaméron: ce manuscrit extraordinaire et hétéroclite, dont il fit usage tout au long de sa carrière, témoigne de ses lectures, de ses annotations, de ses écritures et réécritures, comme allaient le faire bien des siècles plus tard les archives littéraires modernes et contemporaines qui offrent au regard du généticien des documents tels que les carnets de lecture ou les cahiers d'extraits.

Les Frammenti autografi de l'Arioste, que présente Simone Albonico, documentent l'une des dernières phases de l'élaboration du Roland furieux. Édités en 1937 par Santorre Debenedetti dans un ouvrage dont Contini proposa une recension brillante et engagée, ces fragments furent, au $\mathrm{xx}^{\mathrm{e}}$ siècle, au cœur de la naissance (ou renaissance) italienne de la critique des brouillons. Quant à Franco Tomasi, il nous conduit dans l'espace intime et protégé d'un autre grand représentant de la veine épique et chevaleresque italienne, la bibliothèque du Tasse, où le poète aimait se réfugier pour échapper à l'inimitié des courtisans et aux vicissitudes de son existence tourmentée. Tomasi nous montre comment ses livres annotés, matériaux exogénétiques, entrent dans le processus de création de ses chefs-d'œuvre.

11 Au-delà de ces «monuments» de la littérature européenne, la tradition littéraire italienne offre au généticien de nombreux cas d'étude. Ce numéro accorde ainsi une attention particulière à l'œuvre des penseurs de l'Académie des coups de poing, foyer des lumières milanaises. Pierre Musitelli nous présente la correspondance des frères Pietro (1728-1797) et Alessandro Verri (1741-1816) et le système de transmission, d'annotation et de révision de leurs manuscrits qui institue leurs échanges épistolaires trentenaires (1766-1797) en un processus éditorial et une pratique "plurielle» de l'écriture. Gianni Francioni revient, dans son entretien, sur un autre exemple de genèse " en collaboration ", exemplaire des méthodes de travail de l'École de Milan, où les idées des uns et des autres étaient présentées, débattues, développées : celle du célèbre volume Des délits et des peines (1764). L'élaboration de ce texte impliqua plusieurs acteurs: son auteur, bien sûr, Cesare Beccaria (1738-1794), qui rédige le premier manuscrit autographe, mais aussi Pietro Verri, qui corrige et amende le texte à 
plusieurs reprises pour lui donner une tournure juridique et argumentative plus rigoureuse, et enfin le traducteur français, André Morellet, qui restructure profondément le petit libelle philosophique en un véritable «traité ». Voilà un cas de genèse post-éditoriale ${ }^{9}$ particulièrement frappant si l'on pense que, pendant deux siècles, les éditions de cet ouvrage célèbre se conformèrent à l'ordre français établi par Morellet, y compris en Italie! Au moment où s'affirme partout en Europe la figure moderne de l'écrivain, maître de la destinée de ses œuvres, le cas des Délits et des peines invite donc à s'interroger sur la notion d'auctorialité ${ }^{10}$.

12 Celui des Fiancés d'Alessandro Manzoni (1785-1873), présenté par Giulia Raboni, n'est pas moins crucial à plusieurs égards pour la tradition littéraire de l'Italie moderne, dans la mesure où ce roman, rapidement devenu canonique, a offert en partage à la nation naissante "une nouvelle grammaire morale, culturelle et linguistique ». Le mode opératoire de Manzoni, qui élabore sa construction narrative au fil de la plume, en fait un cas emblématique d'écriture à processus et de genèse en mouvement, où les frontières entre les différentes phases rédactionnelles s'estompent jusqu'à disparaître.

13 Avec les Fiancés, dont l'édition définitive paraît en 1842, une coupure symbolique se produit dans la tradition littéraire de la péninsule, comme l'observe Christian Del Vento : le regard que la culture italienne porte sur les manuscrits d'auteur change, on l'a dit, au cours de la seconde moitié du XIX siècle et rejoint progressivement celui des autres traditions littéraires nationales. En témoignent les pages consacrées aux inédits d'Elsa Morante par Monica Zanardo et d'Antonio Tabucchi par Vincenza Perdichizzi : les documents présentés (un avant-texte de La Storia et un court chapitre de la première version du Fil de l'horizon) sont autant d'exemples des processus de remaniement, réécriture et réagencement par lesquels un ouvrage peut trouver sa source dans les impasses d'un projet antérieur, se nourrir de chapitres inaboutis, de versions écartées. Les chemins de la création, qu'un appareil des variantes ne saurait dans de tels cas représenter, s'offrent ici à un passionnant récit de genèse, qui retrace « l'après-coup de l'avant-texte ${ }^{11}$ ». À l'inverse, les écrits d'Antonio Gramsci, que nous évoquons au cours de notre entretien avec Gianni Francioni, conduisent à s'interroger sur le statut des documents avant-textuels en l'absence d'un texte achevé: les Cahiers de prison de l'intellectuel marxiste, véritable "labyrinthe de papier", rassemblent des notes hétérogènes, restées à l'état fluide et magmatique qui précède et virtuellement prélude à la phase de mise en œuvre. Comment représenter et donner à lire cet ensemble fragmentaire, à l'architecture complexe et sinueuse?

$14 \mathrm{Au}$ terme de notre parcours à travers la longue histoire italienne des brouillons d'auteur, les échos de la tradition se sont estompés et les manuscrits ont progressivement perdu leur fonction originelle de garantir l'authenticité des usages linguistiques de leur auteur. C'est désormais la genèse de l'œuvre qui prime. Erri De Luca, dans le double entretien qu'il nous a accordé avec son archiviste Silvia Acocella, fait preuve d'un singulier désintérêt pour ses propres manuscrits (qu'il conserve pourtant), doublé du refus du titre d'«auteur». La notion romantique d'auteurdémiurge s'efface ici devant la résurgence d'une conception classique : celle de l'aède, dépositaire d'un répertoire collectif dont il est le porte-voix. Ses manuscrits doivent alors être envisagés comme le témoignage d'une phase transitoire entre l'écoute et l'écriture, au cours de laquelle l'auteur se fait rédacteur et la création devient épiphanie. Le cas italien s'apparente en fin de compte à un observatoire privilégié pour appréhender, outre la construction de la figure moderne de l'auteur dans ses multiples 
facettes et en amont de ses mutations contemporaines, la naissance et la formation, au fil des siècles, d'une langue et d'une tradition littéraires qui ont puisé leur richesse dans l'étude des brouillons de maîtres.

\section{NOTES}

1. Sur l'ouverture de la critique génétique aux textes antérieurs au XIX siècle, voir Genesis $\mathrm{n}^{\circ} 34$, 2012, [en ligne], et notamment la présentation de N. Ferrand («L'Ancien et le Nouveau Régime des manuscrits de travail », ibid., p. 7-17).

2. M. Motolese et E. Russo (dir.), Autografi dei letterati italiani, Rome, Salerno, 2009 et suiv., 4 vol.

3. P. O. Kristeller (dir.), Iter Italicum. A Finding List of Uncatalogued or Incompletely Catalogued Humanistic Manuscripts of the Renaissance in Italian and Other Libraries, Londres-Leyde, The Warburg Institute-Brill, 1963-1997, 6 vol.

4. Le Rime di Messer Francesco Petrarca estratte da un suo originale..., Rome, Grignani, 1642.

5. Prose di M. Pietro Bembo nelle quali si ragiona della volgar lingua..., Venise, G. Tacuino, 1525.

6. R. Chartier, Culture écrite et Société: l'ordre des livres (XIV ${ }^{e}-X V I I I^{e}$ siècle), Paris, Albin Michel, 1996, p. 69 : «Produit d'une écriture autographe (et non pas copie par un scribe), le "livre d'auteur" doit manifester sans qu'elles soient trahies ou détériorées les intentions qui ont présidé à la composition de l'œuvre. »

7. Voir L. Paolino, « Dans le laboratoire de Pétrarque. Les variantes d'auteur du Chansonnier et des Triomphes ", Arzanà, n 5, 2000, p. 57-77 ; C. Segre, "Critique des variantes et critique génétique », Genesis, n 7, 1995, p. 29-45, et aussi L. Hay, «Défense et illustration de la page », Genesis, n³7, 2013, p. 33-53, [en ligne].

8. Sur les genèses à plusieurs mains, voir N. Donin et D. Ferrer, «Auteur(s) et acteurs de la genèse ", Genesis, n 41, 2015, p. 9 et note 4 notamment, [en ligne].

9. Sur la genèse post-éditoriale, voir Genesis, $\mathrm{n}^{\circ} 44,2017$ : Après le texte. De la réécriture après publication, [en ligne].

10. Sur la notion d'auctorialité, voir J. Stillinger, Multiple Authorship and the Myth of Solitary Genius, New York-Oxford, Oxford University Press, 1991.

11. Voir D. Ferrer, «La toque de Clémentis. Rétroaction et rémanence dans les processus génétiques », Genesis, n 6, 1994, p. 93, [en ligne]. 